\title{
On the Electromagnetic Inverse Scattering Problem
}

\author{
V. H.Weston, J. J. Bowman \& Ergun Ar \\ Communicated by J. MeIXNER
}

\section{Introduction}

Our main purpose is to provide the analyses which we have found to be significant in our investigations of the problem of electromagentic inverse scattering; i.e., the problem where the transmitted field is given and the received fields are measured, and this data is used to discover the nature of the bounded and perfectly conducting object imbedded in the Euclidean 3-space. It is well known that the far field data determines the scattered field everywhere in some domain (e.g. Wilcox [4]) which is easily shown to be exterior to the body. We shall show that, under certain general conditions, the representation for the scattered field is indeed analytic in a region even inside the body.

Our procedure is as follows. In Section 2 we introduce the concept of equivalent sources wherein the scattered field may be trough of as arising from a set of equivalent sources located on or within the boundary of the object. Our approach to the problem is based upon the representation of the scattered field in terms of plane waves. In Sections 3 and 4, an explicit expression for the scattered field, valid in a half space that depends upon the coordinate system, is given in terms of an integral operating on the far scattering field. In Section 5, which is of key importance, we show that the plane wave representation converges even inside the physical boundary under certain conditions, thus establishing the concept that the equivalent sources often may be inside the actual scattering body. Throughout the paper we shall assume for simplicity that the incident field is a plane electromagnetic wave, although the results may be extended to include the case where the primary source is located at a finite distance from the scattering object.

By rotation of axes the integral representation can be used to find the near field everywhere outside the "minimum" convex shape enclosing the equivalent sources. In Sections 6 and 7 we apply our procedure to two specific cases.

\section{The Concept of Equivalent Sources}

The scattered field may be thought of as produced by a set of equivalent sources located on or within the surface of the scattering body. These sources are not necessarily the same as the measurable currents induced, for example, on the surface of a conducting object, but are related to the mathematical representation of the scattered field in the space exterior to the body. As we shall see, there are various methods for obtaining field representations valid everywhere outside the scattering object from a knowledge of the complete radiation pattern of the scattered field. In addition, however, these representations may also be 
analytic in a region inside the scattering surface, in which case the resultant field expression there may be conceived of as being produced by fictitious sources in the absence of the actual body. At points outside the geometrical surface of the body, the field produced by these fictitious sources is identical to the scattered field of the body; the sources are therefore called equivalent sources for the scattered field.

The equivalent sources are not, in general, unique - although they are confined to some finite region of space. The problem of determining the location and extent of this region is fundamental to the inverse scattering investigation and, of course, is intimately connected with the domain of convergence of the mathematical field representation.

The Mie series for scattering by a sphere provides an example in which the formal series solution for the scattered field converges absolutely and uniformly inside as well as outside the body. The scattered field may be conceived of as a superposition of electric and magnetic multipole fields whose sources are located at a single point - the center of the sphere. Upon deletion of this point, the field representation given by the Mie series is analytic everywhere in space.

\section{The Plane Wave Expansion}

A fundamental representation of the electromagnetic field in free space may be obtained as a combination of infinite plane waves whose amplitude factors are given by the far field and whose directions of propagation are, in general, complex. Such a technique has been used for particular direct scattering problems, and modified versions of it appropriate to the high frequency case, have been employed in geometric optics [1].

Consider the electromagentic field produced by a given volume distribution of electric currents $j$ varying harmonically with time $\left(e^{-i \omega t}\right)$ and located in some finite volume $\mathrm{V}$ of free space. The field everywhere in space may be expressed in terms of the vector potential $\boldsymbol{A}$ given by

$$
A(x)=\frac{\mu_{0}}{4 \pi} \int_{V} j\left(x^{\prime}\right) \frac{e^{i k R}}{R} d x^{\prime}
$$

where $R=\left|\boldsymbol{x}-\boldsymbol{x}^{\prime}\right|$, and the far-field distribution has the form

with

$$
A(r, \theta, \phi) \underset{r \rightarrow \infty}{\sim} \frac{e^{i k r}}{r} A_{0}(\theta, \phi)
$$

$$
A_{0}(\theta, \phi)=\frac{\mu_{0}}{4 \pi} \int_{V} j\left(x^{\prime}\right) e^{-i k \cdot x^{\prime}} d x^{\prime}
$$

and

$$
\boldsymbol{k}=k(\sin \theta \cos \phi, \sin \theta \sin \phi, \cos \theta) .
$$

The currents $\boldsymbol{j}$ may again be thought of as equivalent sources for some scattered field or as real sources for some radiation field.

For points exterior to $V$ the Green's function $e^{i k R} / R$ can be expanded into plane waves. We shall employ the well-known integral representation due to 
Weyl [3] (see also Stratton [2]),

$$
\frac{e^{i k R}}{R}=\frac{i k}{2 \pi} \int_{0}^{2 \pi} \int_{0}^{\frac{\pi}{2}-i \infty} e^{i k \cdot\left(x-x^{\prime}\right)} \sin \alpha d \alpha d \beta \quad\left(z \geqq z^{\prime}\right)
$$

where now $k=k(\sin \alpha \cos \beta, \sin \alpha \sin \beta, \cos \alpha)$ is a function of the variables of integration $\alpha$ and $\beta$ running from 0 to $(\pi / 2)-i \infty$ and 0 to $2 \pi$, respectively. It is seen that in this expansion of the spherical wave $e^{i k R} / R$ all possible plane-wave directions within the limits $0 \leqq \beta \leqq 2 \pi, 0 \leqq \alpha \leqq(\pi / 2)$ are included; values of $\alpha$ lying in $(\pi / 2) \leqq \alpha \leqq \pi$ correspond to plane waves traveling in from infinity in the halfspace $z \geqq z^{\prime}$, and are, therefore, excluded. In addition, however, inhomogeneous plane waves with an exponentially decreasing amplitude in the $z$-direction (for $z>z^{\prime}$ ) are included in order to yield the necessary singularity at $R \rightarrow 0$. These waves correspond to that part of the integration path running from $\alpha=(\pi / 2)$ to $\alpha=(\pi / 2)-i \infty$. An alternative representation valid in the half-space $z \leqq z^{\prime}$ may be selecting a different path of integration in the $\alpha$-plane; thus, for example, we may write

$$
\frac{e^{i k R}}{R}=-\frac{i k}{2 \pi} \int_{0}^{2 \pi} \int_{\pi}^{\frac{\pi}{2}+i \infty} e^{i k \cdot\left(x-x^{\prime}\right)} \sin \alpha d \alpha d \beta \quad\left(z \leqq z^{\prime}\right) .
$$

When (3.4) is introduced in (3.1) and the orders or integration interchanged, one obtains for the vector potential $\boldsymbol{A}$ the following result:

$$
\begin{aligned}
\boldsymbol{A}(\boldsymbol{x}) & =\frac{i k}{2 \pi} \frac{\mu_{0}}{4 \pi} \int_{V} j\left(\boldsymbol{x}^{\prime}\right) \int_{0}^{2 \pi} \int_{0}^{\frac{\pi}{2}-i \infty} e^{i \boldsymbol{k} \cdot\left(\boldsymbol{x}-\boldsymbol{x}^{\prime}\right)} \sin \alpha d \alpha d \beta d \boldsymbol{x}^{\prime} \\
& =\frac{i k}{2 \pi} \int_{0}^{2 \pi} \int_{0}^{\frac{\pi}{2}-i \infty} e^{i \boldsymbol{k} \cdot \boldsymbol{x}}\left\{\frac{\mu_{0}}{4 \pi} \int_{V} j\left(x^{\prime}\right) e^{-i k \cdot x^{\prime}} d \boldsymbol{x}^{\prime}\right\} \sin \alpha d \alpha d \beta,
\end{aligned}
$$

and upon recognizing in view of (3.3) that the quantity contained in the braces immediately above is merely $A_{0}(\alpha, \beta)$, one finds

$$
A(x)=\frac{i k}{2 \pi} \int_{0}^{2 \pi} \int_{0}^{\frac{\pi}{2}-i \infty} e^{i k \cdot x} A_{0}(\alpha, \beta) \sin \alpha d \alpha d \beta
$$

provided $\boldsymbol{x}$ lies in the half-space formed by the portion of the $z$-axis above the source volume $V$, that is, $z>z_{\max }^{\prime}$. In this upper half-space, then, (3.7) provides a representation of the near field in terms of the far-field data. For $\boldsymbol{x}$ lying in the lower half-space below the source region, $z<z_{\min }^{\prime}$ we have by virtue of (3.5)

$$
A(x)=-\frac{i k}{2 \pi} \int_{0}^{2 \pi} \int_{\pi}^{\frac{\pi}{2}+i \infty} e^{i k \cdot x} A_{0}(\alpha, \beta) \sin d \alpha d \beta .
$$

The integrals (3.7) and (3.8) together give the field everywhere in space except in the region $z_{\min }^{\prime} \leqq z \leqq z_{\max }^{\prime}$ which sandwiches the sources. It is clear, however, that other paths of integration in the $\alpha$-plane depending on the observation angle $\theta$ may be selected to yield results even within $z_{\min }^{\prime} \leqq z \leqq z_{\max }^{\prime}$, although the source 
region must still be excluded. Choosing other paths of integration is tantamount to rotating the reference axes.

Finally, it should be mentioned that the plane-wave expansion of the near field in terms of the far field could have been derived by starting with a surface integral representation of the vector potential. For example, in the case of a perfectly conducting body, the vector potential for the scattered field is given by

$$
\boldsymbol{A}^{\mathrm{sc}}(\boldsymbol{x})=\frac{\mu_{0}}{4 \pi} \int_{S}(\boldsymbol{n} \wedge \boldsymbol{H}) \frac{e^{i k R}}{R} d S^{\prime}
$$

where $S$ is the exterior surface of the scattering body and $\boldsymbol{H}$ is the total magnetic field generated on the surface. Introducing (3.4) into (3.9) and interchanging the orders of integration, one finds

$$
A^{\mathrm{sc}}(\boldsymbol{x})=\frac{i k}{2 \pi} \int_{0}^{2 \pi} \int_{0}^{\frac{\pi}{2}-i \infty} e^{i \boldsymbol{k} \cdot \boldsymbol{x}} A_{0}^{\mathrm{sc}}(\alpha, \beta) \sin \alpha d \alpha d \beta
$$

provided $\boldsymbol{x}$ lies in the half-space formed by the portion of the $z$ axis above the scattering surface $S$. The last condition may be too restrictive, however, and the integral in (3.10) may be convergent for points $x$ lying inside the scattering surface, where it will represent the field produced by an equivalent source. This very important observation is considered in Section 5 where the domain of convergence is extended into smooth convex sections of a perfectly conducting scattering surface.

The electromagnetic field is derived from the vector potential by means of the relations

$$
\boldsymbol{H}=\frac{1}{\mu_{0}} \nabla \wedge \boldsymbol{A}, \quad \boldsymbol{E}=\frac{i}{\varepsilon_{0} \mu_{0} \omega} \nabla \wedge \nabla \wedge \boldsymbol{A} .
$$

In the far zone these equations give

$$
\begin{aligned}
& \boldsymbol{H}(r, \theta, \phi) \sim \frac{e^{i k r}}{r} H_{0}(\theta, \phi)=\frac{e^{i k r}}{r}\left(\frac{i}{\mu_{0}}\right) \boldsymbol{k} \wedge A_{0}(\theta, \phi), \\
& \boldsymbol{E}(r, \theta, \phi) \sim \frac{e^{i k r}}{r} E_{0}(\theta, \phi)=\frac{e^{i k r}}{r}\left(\frac{i}{\varepsilon_{0} \mu_{0} \omega}\right) k \wedge k \wedge A_{0}(\theta, \phi) .
\end{aligned}
$$

When these relations are applied to the integral representation (3.7) of the vector potential, one finds

$$
\begin{aligned}
\boldsymbol{H}(\boldsymbol{x}) & =\frac{i k}{2 \pi} \int_{0}^{2 \pi} \int_{0}^{\frac{\pi}{2}-i \infty} e^{i \boldsymbol{k} \cdot \boldsymbol{x}}\left(\frac{i}{\mu_{0}}\right) k \wedge A_{0}(\alpha, \beta) \sin \alpha d \alpha d \beta \\
& =\frac{i k}{2 \pi} \int_{0}^{2 \pi} \int_{0}^{\frac{\pi}{2}-i \infty} e^{i k \cdot x} H_{0}(\alpha, \beta) \sin \alpha d \alpha d \beta,
\end{aligned}
$$

and similarly for the electric field

$$
\boldsymbol{E}(\boldsymbol{x})=\frac{i k}{2 \pi} \int_{0}^{2 \pi} \int_{0}^{\frac{\pi}{2}-i \infty} e^{i k \cdot x} E_{0}(\alpha, \beta) \sin \alpha d \alpha d \beta .
$$

The near field may thus be represented directly in terms of the electromagnetic fields in the far zone. 


\section{Analytic Continuation}

The field $\boldsymbol{H}_{0}(\theta, \phi)$ in the far zone is measurable only for real values of $\theta, \phi$ in the ranges $0 \leqq \theta \leqq \pi, 0 \leqq \phi \leqq 2 \pi$. However, in order to obtain the near field by means of the integral representation discussed in the previous section, it is necessary to know $H_{0}(\alpha, \phi)$ where $\alpha=\theta+i \xi$. Therefore, we need an extension into the complex $\alpha$-plane based upon the measured quantity $\boldsymbol{H}_{0}(\theta, \phi)$.

Now $\boldsymbol{H}_{0}(\theta, \phi)$ is immediately known for the range $-\pi \leqq \theta \leqq \pi$. This follows from the definition

$$
\boldsymbol{H}_{0}(\theta, \phi)=\frac{i}{4 \pi} \int_{\boldsymbol{V}} \boldsymbol{k} \wedge \boldsymbol{j}\left(x^{\prime}\right) e^{-i \boldsymbol{k} \cdot \boldsymbol{x}^{\prime}} d \boldsymbol{x}^{\prime}
$$

and the fact that $\boldsymbol{k}$ as a function of $\theta$ and $\phi$ satisfies

hence

$$
\boldsymbol{k}(-\theta, \phi)=\boldsymbol{k}(\theta, \phi \pm \pi)
$$

$$
\boldsymbol{H}_{0}(-\theta, \phi)=\boldsymbol{H}_{0}(\theta, \phi \pm \pi) \text {. }
$$

In addition, $\boldsymbol{H}_{0}$ is periodic with period $2 \pi$ in both $\theta$ and $\phi$.

To obtain an expression for $\boldsymbol{H}(\alpha, \phi)$ in the complex $\alpha$-plane, we observe from (4.1) that $\boldsymbol{H}_{0}(\alpha, \phi)$ is a harmonic function in the variables $\theta$ and $\xi$; that is

$$
\left(\frac{\partial^{2}}{\partial \theta^{2}}+\frac{\partial^{2}}{\partial \xi^{2}}\right) H_{0}(\theta+i \xi, \phi)=0 \text {. }
$$

Therefore, $\boldsymbol{H}_{0}(\alpha, \phi)$ may be expressed formally as

$$
H_{0}(\alpha, \phi)=\sum_{n=-\infty}^{\infty} a_{n} e^{i n(\theta+i \xi)}
$$

where the coefficients $a_{n}$ are derived by means of the relation

$$
\boldsymbol{a}_{n}=\frac{1}{2 \pi} \int_{-\pi}^{\pi} e^{-i n \theta^{\prime}} \boldsymbol{H}_{0}\left(\theta^{\prime}, \phi\right) d \theta^{\prime}
$$

This provides an extension into the complex $\alpha$-plane. The series (4.5) may be partially summed and put into the following form:

$\boldsymbol{H}_{0}(\alpha, \phi)=\frac{1}{2 \pi} \int_{-\pi}^{\pi} \frac{e^{i(\theta+i \xi)} \boldsymbol{H}_{0}\left(\theta^{\prime}, \phi\right)}{e^{i(\theta+i \xi)}-e^{i \theta^{\prime}}} d \theta^{\prime}+\frac{1}{2 \pi} \sum_{n=1}^{\infty} e^{i n(\theta+i \xi)} \int_{-\pi}^{\pi} \boldsymbol{H}_{0}\left(\theta^{\prime}, \phi\right) e^{i n \theta^{\prime}} d \theta^{\prime}$

for $\xi<0$, and

$\boldsymbol{H}_{0}(\alpha, \phi)=\frac{1}{2 \pi} \int_{-\pi}^{\pi} \frac{e^{i \theta^{\prime}} \boldsymbol{H}_{0}\left(\theta^{\prime}, \phi\right)}{e^{i \theta^{\prime}}-e^{i(\theta+i \xi)}} d \theta^{\prime}+\frac{1}{2 \pi} \sum_{n=1}^{\infty} e^{-i n(\theta+i \xi)} \int_{-\pi}^{\pi} H_{0}\left(\theta^{\prime}, \phi\right) e^{i n \theta^{\prime}} d \theta^{\prime}$ for $\xi>0$.

To investigate the convergence of the series (4.5) we examine the behavior of $a_{n}$ as $n \rightarrow \infty$. Now

$$
\begin{aligned}
a_{n} & =\frac{1}{2 \pi} \int_{-\pi}^{\pi} e^{-i n \theta}\left\{\frac{i}{4 \pi} \int_{V} k \wedge j\left(x^{\prime}\right) e^{-i k \cdot x^{\prime}} d x^{\prime}\right\} d \theta \\
& =\frac{-i}{8 \pi^{2}} \int_{V} j\left(x^{\prime}\right) \wedge \int_{-\pi}^{\pi} k e^{-i n \theta-i k \cdot x^{\prime}} d \theta d x^{\prime}
\end{aligned}
$$


where

Write $\boldsymbol{k}$ in the form

$$
\boldsymbol{k}=k(\sin \theta \cos \phi, \sin \theta \sin \phi, \cos \theta)
$$

with

$$
k=\frac{k}{2}\left(e^{i \theta} t+e^{-i \theta} t^{*}\right)
$$

then

$$
t=(-i \cos \phi,-i \sin \phi, 1)
$$

$a_{n}=\frac{-i k}{16 \pi^{2}} \int_{V} j\left(x^{\prime}\right) \wedge\left\{t \int_{-\pi}^{\pi} e^{-i(n-1) \theta-i k \cdot x^{\prime}} d \theta+t^{*} \int_{-\pi}^{\pi} e^{-i(n+1) \theta-i k \cdot x^{\prime}} d \theta\right\} d x^{\prime}$.

But $\boldsymbol{k} \cdot \boldsymbol{x}^{\prime}$ may be written in the form

where

$$
\begin{aligned}
\boldsymbol{k} \cdot \boldsymbol{x}^{\prime} & =k r^{\prime}\left[\cos \theta \cos \theta^{\prime}+\sin \theta \sin \theta^{\prime} \cos \left(\phi-\phi^{\prime}\right)\right] \\
& =k r^{\prime} \rho \cos (\theta-\psi),
\end{aligned}
$$

$$
\begin{aligned}
\rho^{2} & =\cos ^{2} \theta^{\prime}+\sin ^{2} \theta^{\prime} \cos ^{2}\left(\phi-\phi^{\prime}\right), \\
\tan \psi & =\tan \theta^{\prime} \cos \left(\phi-\phi^{\prime}\right),
\end{aligned}
$$

and, in view of the integral representation

$$
e^{-i m \psi} J_{m}\left(k r^{\prime} \rho\right)=\frac{i^{n}}{2 \pi} \int_{-\pi}^{\pi} e^{-i m \theta-i k r^{\prime} \rho \cos (\theta-\psi)} d \theta
$$

we have the closed form expression

$$
a_{n}=\frac{(-i)^{n} k}{8 \pi} \int_{V} j\left(x^{\prime}\right) \wedge\left\{t e^{i \psi} J_{n-1}\left(k r^{\prime} \rho\right)-t^{*} e^{-i \psi} J_{n+1}\left(k r^{\prime} \rho\right)\right\} e^{-i n \psi} d x^{\prime}
$$

As $n$ tends to infinity the dominant contribution is due to the first term within the braces in the integrand; thus, employing the large order asymptotic expansion for the Bessel function, we have

and

$$
a_{n \rightarrow \infty} \sim \frac{(-i)^{n} k}{8 \pi \Gamma(n)} \int_{V} j\left(x^{\prime}\right) \wedge t\left(\frac{k r^{\prime} \rho}{2} e^{-i \psi}\right)^{n-1} d x^{\prime},
$$

$$
\left|a_{n}\right|_{n \rightarrow \infty} \leqq \frac{\text { Constant }}{\Gamma(n)}\left(\frac{k R}{2}\right)^{n-1}
$$

where $R$ represents the maximum value of $r^{\prime}$. A similar results holds for $\left|a_{-n}\right|$. The convergence of the series (4.5) is therefore secured for all $\alpha=\theta+i \xi$ because of the gamma function in the denominator of (4.19).

It should be pointed out that although the coefficients $a_{n}$ can be obtained from measurements of $\boldsymbol{H}_{0}(\theta, \phi)$, they can be obtained only within some accuracy which becomes worse the larger $n$ is. The formal analytic continuation (4.5) is therefore undetermined when based upon a finite number of measurements. On the other hand, the far field quantity $H_{0}(\theta, \phi)$ arises from a current distribution located in a finite portion of space. Therefore the coefficients $a_{n}$ decrease to zero very strongly as $|n| \rightarrow \infty$ (see 4.19) and this is all that is needed. 


\section{The Validity of the Field Representation Interior to the Scatterer}

We recall that the vector potential $\boldsymbol{A}(\boldsymbol{x})$ can be represented as

$$
\boldsymbol{A}(\boldsymbol{x})=\frac{i k}{2 \pi} \int_{0}^{2 \pi} \int_{0}^{\frac{\pi}{2}-i \infty} e^{i \boldsymbol{k} \cdot \boldsymbol{x}} \boldsymbol{A}_{0}(\alpha, \beta) \sin \alpha d \alpha d \beta
$$

provided the position vector $x$ lies in the half-space $z>z_{\max }$, where the scattering body is assumed contained in the slab $z_{\max }>z>z_{\min }$ with the planes $z=z_{\max }$ and $z=z_{\min }$ tangent to the body. Similarly, the representation given in (3.8) is valid for the half-space $z<z_{\min }$. Upon rotation of the coordinate system and use of the

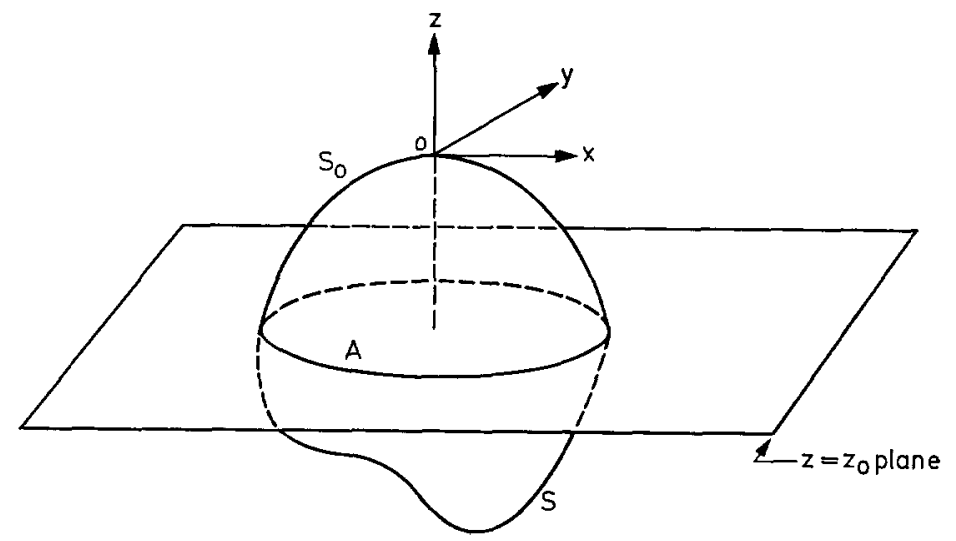

Fig. 1

appropriate representations holding in the new half-spaces $z^{\prime}>z_{\max }^{\prime}$ and $z^{\prime}<z_{\min }^{\prime}$, one can obtain the scattered field in those portions of the slab $z_{\min }<z<z_{\max }$ lying in the half-spaces $z>z_{\max }^{\prime}$ and $z<z_{\min }^{\prime}$. In this way the scattered field is obtained for every point outside the minimum convex surface enclosing the body.

However, it will be shown here that for certain perfectly conducting bodies the plane wave representation (5.1) is convergent in a larger half-space $z>z^{*}$ where $z_{\min }<z^{*}<z_{\max }$, thus implying that the representation is valid part way inside the body. In such an interior portion of the body the representation no longer represents the true scattered field since for a conducting surface the total field inside is zero. As pointed out earlier, such a representation can be conceived of as being produced by fictitious sources (equivalent currents) inside the body. On the other hand, at points in the slab $z^{*}<z<z_{\max }$ which are outside the scattering surface, the representation still represents the scattered field. A general algorithm for computing the value of $z^{*}$ is contained in the proof of the following theorem.

Theorem 5.1. Let an arbitrary plane $z=z_{0}$ intersect a perfectly conducting scattering surface $S$ and denote the portion of $S$ above this plane by $S_{0}$. If $S_{0}$ has the following properties:

(i) $S_{0}$ has the representation $z=f(x, y)$ for $x, y$ in the domain $A$, where $A$ is the domain in the $z_{0}$-plane formed by the points interior to the intersection of $S$ and the $z_{0}$-plane (see Fig. 1), 
(ii) $S_{0}$ is convex,

(iii) $f$ is analytic in the domain $A$,

then the representation (5.1) converges and is analytic for $z>z^{*}$, where $z^{*}$ lies in the interval $\left(z_{\min }, z_{\max }\right)$ and is determined by $f(x, y)$.

Proof. We begin with the expression for the far-zone potential,

$$
\boldsymbol{A}_{0}(\alpha, \beta)=\frac{\mu_{0}}{4 \pi} \int_{S}(\hat{\boldsymbol{n}} \wedge \boldsymbol{H}) e^{-i \boldsymbol{k} \cdot \boldsymbol{x}} d S, \quad \boldsymbol{x} \in S
$$

where $\boldsymbol{H}$ is the total magnetic field generated on the surface $S$ by the incident plane electromagnetic wave and where

$$
\boldsymbol{k}=k(\sin \alpha \cos \beta, \sin \alpha \sin \beta, \cos \alpha) .
$$

We must investigate the behavior of $\boldsymbol{A}_{0}(\alpha, \beta)$ near the endpoint $\alpha=(\pi / 2)-i \infty$. Toward this end we set $\alpha=(\pi / 2)-i t$, so that

$$
\boldsymbol{k}=k(\cosh t \cos \beta, \cosh t \sin \beta, i \sinh t),
$$

and study the asymptotic behavior of the integral (5.2) for $t \rightarrow \infty$. To simplify the analysis, choose the coordinate system so that the origin is on the surface $S$ with the positive $z$ axis normal to $S$ (pointing outwards) and orient the coordinate system so as to have

$$
\boldsymbol{k} \cdot \boldsymbol{x}=k(x \cosh t+i z \sinh t) .
$$

Then, as $t \rightarrow \infty$, equation (5.2) reduces to

$$
\boldsymbol{A}_{0}(\alpha, \beta) \sim \frac{\mu_{0}}{4 \pi} \boldsymbol{I}(\alpha, \beta)+O\left(e^{k z_{0} \sinh t}\right)
$$

where

$$
I(\alpha, \beta)=\int_{S_{0}}(\hat{n} \wedge H) e^{-i k \cdot x} d S, \quad x \in S_{0}
$$

and $z_{0}<0$ due to our choice of coordinates.

Since

$$
\hat{n}=\left(-\frac{\partial f}{\partial x},-\frac{\partial f}{\partial y}, 1\right)\left[1+\left(\frac{\partial f}{\partial x}\right)^{2}+\left(\frac{\partial f}{\partial y}\right)^{2}\right]^{-\frac{1}{2}},
$$

we can write (5.7) as

$$
\boldsymbol{I}=\iint_{\boldsymbol{A}} \exp \{-i k(x \cosh t+i f \sinh t)\}\left(-\frac{\partial f}{\partial x} \hat{\boldsymbol{x}}-\frac{\partial f}{\partial y} \hat{y}+\hat{z}\right) \wedge \boldsymbol{H} d x d y
$$

where $A$ is the projected area of $S_{0}$ on the plane $z=z_{0}$. We perform the $y$ integration first. For $t \rightarrow \infty$ the dominant contribution comes from the neighborhood of the point $y_{0}$, where

$$
\left(\frac{\partial f}{\partial y}\right)_{y_{0}}=0
$$

and by means of a saddle point integration we have

$$
I \sim \sqrt{\frac{2 \pi}{k \sinh t}} \int_{x_{1}}^{x_{2}} \exp \left\{-i k\left[x \cosh t+i f\left(x, y_{0}\right) \sinh t\right]\right\} \boldsymbol{B} d x
$$


with $\boldsymbol{B}$ defined as

$$
\boldsymbol{B}=\left[\left(-\frac{\partial f}{\partial x} \hat{\boldsymbol{x}}-\frac{\partial f}{\partial y} \hat{\boldsymbol{y}}+\hat{z}\right) \wedge \boldsymbol{H}\right]_{y_{0}}\left(-\frac{\partial^{2} f}{\partial y^{2}}\right)_{y_{0}}^{-\frac{1}{2}} .
$$
Note that because of the assumptions (i) to (iii) we have $\left(-\frac{\partial^{2} f}{\partial y^{2}}\right)_{y_{0}}>0$ and there
is only one such point $y_{0}$.

Let us cut the surface by the plane $x=x^{\prime}\left(x_{1}<x^{\prime}<x_{2}\right)$ as shown in Fig. 2. For $z>z_{0}, x_{1}<x^{\prime}<x_{2}$, the function $f\left(x^{\prime}, y\right)$ is monotonically increasing if $y<y_{0}$, and it is monotonically decreasing if $y>y_{0}$. It has a maximum at $y=y_{0}$. This holds
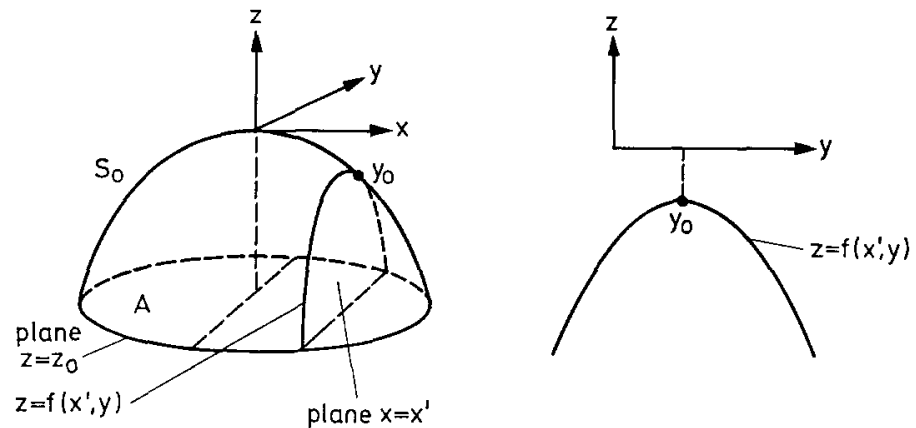

Fig. 2

for all the curves determined by planes $x=x^{\prime}\left(x_{1}<x^{\prime}<x_{2}\right)$. Since the analysis will have to hold for all angles $\beta$, these observations must remain valid for all cuts parallel to the $z$-axis of the surface $S_{0}$. Thus the surface $S_{0}, z=f(x, y)$, has a maximum at the origin and monotonically decreases away from the origin. It may appear that the dominant contribution to (5.11) arises from the point $z=0$ because of the term $\exp \left[k f\left(x, y_{0}\right) \sinh t\right]$. However, the remaining term $\exp [-i k x \cosh t]$ is a rapidly varying function and in the limit when $t \rightarrow \infty$ this can negate the contribution of the integral in the neighborhood of $x=0$. In order to evaluate properly the integral (5.18) we proceed as follows. We define the complex plane $w=x+i v$. Because of our assumptions (i) to (iii) about the surface $S_{0}$, we have

$$
z=f(x, y)=\sum_{m+n=2} a_{m n} x^{m} y^{n} \quad(m, n \text { positive integers })
$$

The function $f\left(w, y_{0}\right)$ is thus analytic in some domain $D_{1}$ which contains the line segment $x_{1} \leqq x \leqq x_{2}$. Assume that the vector function $\boldsymbol{B}\left(w, y_{0}\right)$ is also analytic in a domain $D_{2}$; this domain also must contain the segment $x_{1} \leqq x \leqq x_{2}$. We see that analyticity of $\boldsymbol{B}$ presupposes the analyticity of $\boldsymbol{H}$ on $S_{0}$, and with assumptions (i) to (iii) the analyticity of $\boldsymbol{H}$ can be demonstrated (see Appendix).

Now write the expression (5.11) in the form

$$
I \sim \sqrt{\frac{2 \pi}{k \sinh t}} \int_{C} \exp \left[-i k w \cosh t+k f\left(w, y_{0}\right) \sinh t\right] \boldsymbol{B}\left(w, y_{0}\right) d w,
$$


where the contour $C$ lies in the domain $D=D_{1} \cap D_{2}$, with end-points $w_{1}=x_{1}$, $w_{2}=x_{2}$. The contour $C$ will consist of three parts: the line segment $x_{1} \leqq x \leqq \tilde{x}_{1}$, the contour $C_{1}$ with end-points $\tilde{x}_{1}$ and $\tilde{x}_{2}$, and the line segment $\tilde{x}_{2} \leqq x \leqq x_{2}$, (see Fig. 3). Since

$$
\begin{aligned}
\operatorname{Re}\{ & \left.-i k w \cosh t+k f\left(w, y_{0}\right) \sinh t\right\} \\
& =\operatorname{Re}\{-i k(x+i v) \cosh t\}+\operatorname{Re}\left\{k f\left(w, y_{0}\right) \sinh t\right\} \\
& =k \sinh t\left\{v \operatorname{coth} t+\operatorname{Re}\left[f\left(w, y_{0}\right)\right]\right\},
\end{aligned}
$$

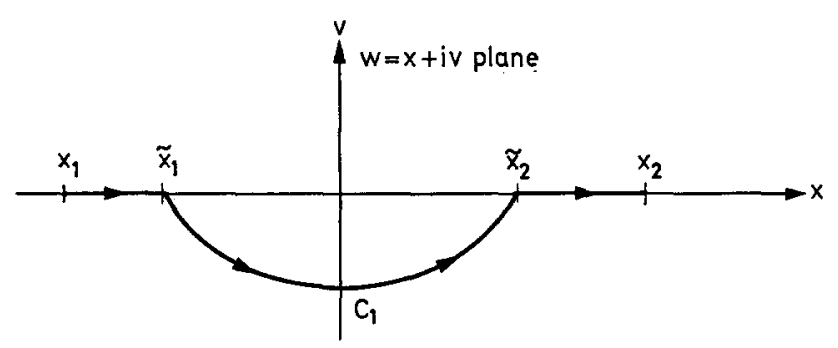

Fig. 3

we shall require the contour $C_{1}$ to have the property that for $w \in C_{1}$

$$
\operatorname{Re}\left\{f\left(w, y_{0}\right)\right\}+v \operatorname{coth} t=f\left(\tilde{x}_{1}, y_{0}\right)=f\left(\tilde{x}_{2}, y_{0}\right) \text {. }
$$

Also, since

$\operatorname{Im}\left\{-i k w \cosh t+k f\left(w, y_{0}\right) \sinh t\right\}=k\left\{-x \cosh t+\sinh t \operatorname{Im}\left[f\left(w, y_{0}\right)\right]\right\}$,

we see that the contribution of contour $C_{1}$ to the integral (5.13) has the asymptotic form for $t \rightarrow \infty$ :

$$
\begin{aligned}
\boldsymbol{I}_{C_{1}} \sim \sqrt{\frac{2 \pi}{k \sinh t}} \cdot \exp \left\{k f\left(\tilde{x}_{1}, y_{0}\right) \sinh t\right\} \\
\cdot \int_{C_{1}} \exp \left\{i k\left[-x \cosh t+\operatorname{Im} f\left(w, y_{0}\right) \sinh t\right]\right\} \boldsymbol{B}(w) d w .
\end{aligned}
$$

But $f\left(|x|, y_{0}\right)$ is a monotonic decreasing function for increasing $|x|$ in the interval $x_{1}<x<x_{2}$; therefore, the dominant contributions of the remaining line integrals arise from the end points $\tilde{x}_{1}, \tilde{x}_{2}$, and have the asymptotic behavior $O\left[\exp k f\left(\tilde{x}_{1}, y_{0}\right) \sinh t\right]$. Thus, in view of (5.17), the complete integral $I$ is of this order.

The quantities $\tilde{x}_{1}$ and $\tilde{x}_{2}$ are chosen such that $f\left(\tilde{x}_{1}, y_{0}\right)$ assumes its minimum value subject to the restraint that $C_{1}+\overline{\tilde{x}}_{1} \tilde{x}_{2}$ is a closed curve in the domain $D$. If the function $i w \operatorname{coth} t+f\left(w, y_{0}\right)$ has a saddle point at $w_{0}=x_{0}+i v_{0}$ then we cannot have a closed curve when $\tilde{x}_{2}>\tilde{x}_{2}^{0}$ (see Fig. 4), where $\tilde{x}_{2}^{0}$ is defined by

$$
\operatorname{Re}\left\{f\left(w_{0}, y_{0}\right)\right\}+v_{0} \cosh t=f\left(\tilde{x}_{2}^{0}, y_{0}\right)
$$


In this case the integral $I$ is of the order $\exp \left(k z^{*} \sinh t\right)$, where $z^{*}=f\left(\tilde{x}_{2}^{0}, y_{0}\right)$. Otherwise, when $\tilde{x}_{2}^{0}>x_{2}$, or when there is no saddle point, $z^{*}=\max \left(f\left(x_{2}, y_{0}\right)\right.$, $\left.f\left(x_{1}, y_{0}\right)\right)=z_{0}$. From (5.6) it follows that

$$
A_{0}(\alpha, \beta)=O\left[\exp k z^{*} \sinh t\right]
$$

and therefore that the representation (5.1) converges and is analytic for $z>z^{*}$, where $z^{*}<0$.

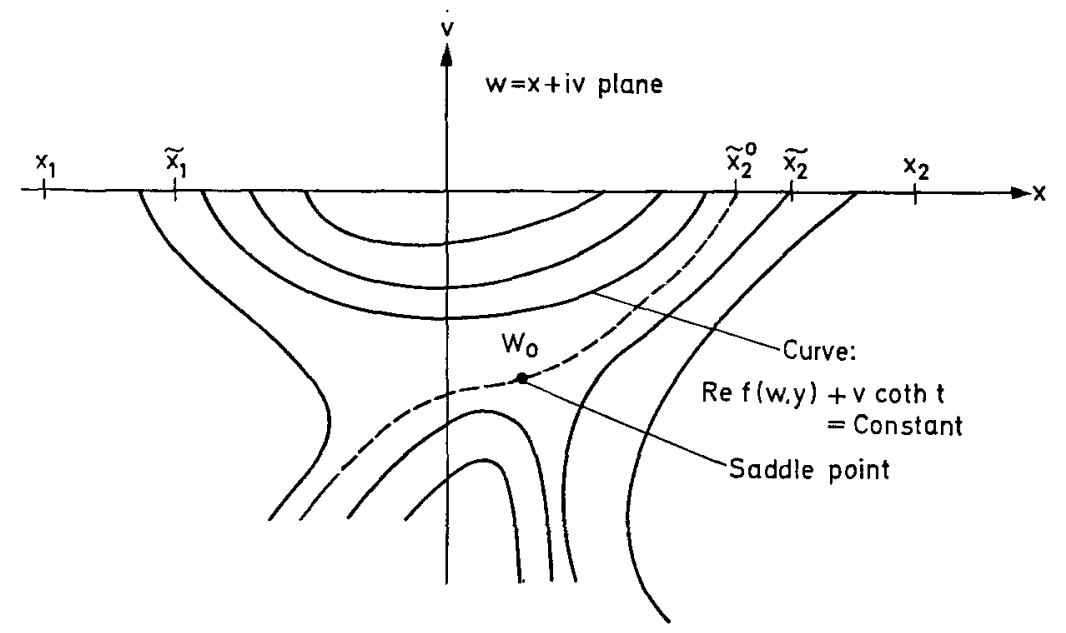

Fig. 4

\section{Application: Elliptic Paraboloid}

Consider the elliptic paraboloid given by

$$
z=-a x^{2}-b x y-c y^{2}=f(x, y), \quad\left(z_{0}=-\infty\right)
$$

whose principal radii of curvature $R_{1}$ and $R_{2}$ are obtained from

$$
2(a+c)=1 / R_{1}+1 / R_{2}, \quad 2 \sqrt{(a-c)^{2}+b^{2}}=1 / R_{1}-1 / R_{2} .
$$

The stationary point $y_{0}$ [cf. equation (5.10)] is given by

and for $y=y_{0}$ we have

$$
-b x-2 c y_{0}=0
$$

$$
f\left(x, y_{0}\right)=-\frac{x^{2}}{4 c}\left[4 a c-b^{2}\right]
$$

For this surface $x_{1}=-\infty$ and $x_{2}=\infty$. We need to consider the possible stationary points of the function

From

$$
-i w \operatorname{coth} t+f\left(w, y_{0}\right), \quad \text { for } t \rightarrow \infty .
$$

$$
\lim _{t \rightarrow \infty} \frac{\partial}{\partial w}\left[-i w \operatorname{coth} t+f\left(w, y_{0}\right)\right]=\lim _{t \rightarrow \infty}\left[-i \operatorname{coth} t-\frac{w}{2 c}\left(4 a c-b^{2}\right)\right]=0,
$$


we see that the stationary point is at $w=w_{0}$, where

$$
w_{0}=\frac{-2 c i}{\left(4 a c-b^{2}\right)} \text {. }
$$

Thus the value of $\tilde{x}_{2}^{0}$ to be taken is given by [cf. equation (5.18)]

that is,

$$
f\left(\tilde{x}_{2}^{0}, y_{0}\right)=\operatorname{Re}\left\{f\left(w_{0}, y_{0}\right)\right\}+v_{0}, \quad \text { for } t \rightarrow \infty,
$$

$$
-\frac{\left(\tilde{x}_{2}^{0}\right)^{2}}{4 c}\left(4 a c-b^{2}\right)=\frac{c}{4 a c-b^{2}}-\frac{2 c}{4 a c-b^{2}}=-\frac{c}{4 a c-b^{2}},
$$

or

$$
\left(\tilde{x}_{2}^{0}\right)^{2}=\frac{4 c^{2}}{\left(4 a c-b^{2}\right)^{2}}
$$

Therefore, in this case we find that

$$
f\left(\tilde{x}_{2}^{0}, y_{0}\right)=-\frac{c}{4 a c-b^{2}} .
$$

From (6.1) and (6.2) we observe

$$
R_{1} R_{2}=\frac{1}{4 a c-b^{2}}
$$

and the curvature of the curve $z=f(x, y), x=0$ is

If we let

$$
\frac{-2 c}{\left(1+4 c^{2} y^{2}\right)^{3 / 2}}
$$

$$
-\frac{1}{R_{y}}=\left.\frac{-2 c}{\left(1+4 c^{2} y^{2}\right)^{3 / 2}}\right|_{y=0}=-2 c,
$$

then we can write (6.9) as

$$
f\left(\tilde{x}_{2}^{0}, y_{0}\right)=-\frac{R_{1} R_{2}}{2 R_{y}} .
$$

Now we go back and from (5.17) observe that the integral $I$ has the dominant asymptotic behavior

$$
I \sim O\left[\exp \left(-k \cdot \sinh t \cdot \frac{R_{1} R_{2}}{2 R_{y}}\right)\right] .
$$

Note that in the original expression [equations (5.2), (5.3)] the angle $\beta$ occurred. The coordinate system was rotated to remove the dependence on $\beta$, so that the proper asymptotic behavior for all angles $\beta$ requires a rotation of the coordinate system. This effectively changes the value of $R_{y}$. It goes from $R_{1}$ to $R_{2}$ where $R_{1}$ is the minimum radius of curvature. Therefore,

$$
I \sim \exp \left(-k \sinh t \cdot \frac{R_{\min }}{2}\right)
$$


From (5.6) and (6.15) we see that $e^{i k \cdot x} A_{0} \rightarrow 0$ exponentially as

$$
\alpha \rightarrow \frac{\pi}{2}-i \infty
$$

for $z>z^{*}$ where

$$
z^{*}=-\frac{R_{\min }}{2} .
$$

The plane wave representation (5.1) is thus convergent not only for $z>0$ but also for the larger half-space $z>-(1 / 2) R_{\min }$.

\section{Application: Spheroid}

We now consider a body of revolution, and take the $z$-axis to be the axis of revolution. In this case the orientation of the $x, y$ axes does not matter.

The surface is given by

$$
\begin{gathered}
z=f(x, y)=-b+b \sqrt{1-\frac{p^{2}}{a^{2}}}, \\
p^{2}=x^{2}+y^{2}, \quad p<a \quad\left(z_{0}=-a\right) .
\end{gathered}
$$

The stationary point $y=y_{0}$ for which $\frac{\partial f}{\partial y}=0$ is $y_{0}=0$, and we have

$$
f\left(w, y_{0}\right)=-b+b \sqrt{1-\frac{w^{2}}{a^{2}}} .
$$

This function will be taken to have cuts along the $x$-axis, $x>a$, and $x<-a$. Let $w_{0}$ be the stationary point of

This is given by

$$
-i w+f\left(w, y_{0}\right) \quad(t=\infty)
$$

$$
i=\frac{\partial f}{\partial w}=-\frac{b w}{a \sqrt{a^{2}-w^{2}}}
$$

thus

provided that $b>a$.

$$
w_{0}=-\frac{i a^{2}}{\sqrt{b^{2}-a_{2}^{2}}}
$$

From equation (5.18), for $t=\infty$, we determine $\tilde{x}_{2}^{0}$ so that

$$
\operatorname{Re}\left\{,\left(w_{0}, y_{0}\right)\right\}+v_{0}=f\left(\tilde{x}_{2}^{0}, y_{0}\right) \text {. }
$$

Thus, after a short calculation we obtain

$$
\tilde{x}_{2}^{0}=\frac{a^{2}}{b}=R_{0}
$$

where $R_{0}$ is the radius of curvature at the tip. We find that

$$
f\left(\tilde{x}_{2}^{0}, y_{0}\right)=-b+b \sqrt{1-\frac{\left(\tilde{x}_{2}^{0}\right)^{2}}{a^{2}}}=-b+\sqrt{b^{2}-a^{2}} .
$$


Thus the asymptotic behavior of $I$ for $t \rightarrow \infty$ is given by

$$
I \sim \exp \left\{-k\left[\sinh t b-\sqrt{b^{2}-a^{2}}\right]\right\} .
$$

This means that the region of convergence of the plane wave expansion holds for $z>z^{*}$ with

$$
z^{*}=-b+\sqrt{b^{2}-\bar{a}^{2}}, \quad(b>a) .
$$

This plane goes through the focus of the spheroid as shown in Fig. 5. Finally, we note that the result for the sphere follows immediately if we put $b=a+\varepsilon$ and take

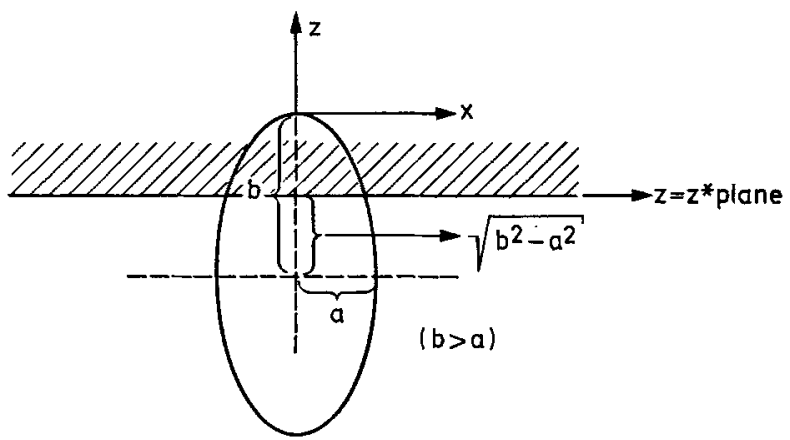

Fig. 5

$\varepsilon>0$ to be arbitrarily small. In this regard, recall that the field representation given by the Mie series solution is analytic everywhere in space upon deletion of the center point of the sphere.

\section{Appendix}

We now show that from the assumptions (i), (ii), (iii) in Theorem 5.1, it follows that the field $(\boldsymbol{E}, \boldsymbol{H})$ is analytic on the perfectly conducting surface $S$.

Let $\left(\boldsymbol{E}^{i}, \boldsymbol{H}^{i}\right)$ denote the incident field, which we assume to be a plane wave, and $\left(\boldsymbol{E}^{s}, \boldsymbol{H}^{s}\right)$ the scattered field. Since $\boldsymbol{E}^{i}$ is analytic everywhere, and $S$ is assummed to be analytic, from

it follows that

$$
\left.\hat{\boldsymbol{n}} \wedge \boldsymbol{E}\right|_{s}=\left.\hat{\boldsymbol{n}} \wedge\left(\boldsymbol{E}^{i}+\boldsymbol{E}^{s}\right)\right|_{s}=0
$$

$$
\widehat{\boldsymbol{n}}(x, y, f(x, y)) \wedge \boldsymbol{E}^{s}(x, y, f(x, y))=\hat{\boldsymbol{n}}(x, y) \wedge \boldsymbol{E}^{s}(x, y)
$$

is analytic; where $x, y \in A$ and $A$ is domain of analyticity of $f$ on the $x$-y-plane. From the Maxwell equation

it follows that

$$
\nabla \wedge H(x, y, z)=-i \omega \varepsilon_{0} E(x, y, z)
$$

$$
\hat{n}(x, y, f(x, y)) \wedge[\nabla \wedge H(x, y, z)]_{z \in S}=-i \omega \varepsilon_{0} n(x, y) \wedge E(x, y), \quad x, y \in A ;
$$

that is,

$$
\hat{\boldsymbol{n}}(x, y) \wedge[\nabla \wedge \boldsymbol{H}(x, y, z)]_{z \in S}
$$


is analytic on $A$. But the components $n_{x}, n_{y}, n_{z}$ of $\hat{\boldsymbol{n}}(x, y f(x, y))$ are analytic on $A$, and if we denote the components of $\boldsymbol{H}$ on $S$ by $H_{x}(x, y), H_{y}(x, y), H_{z}(x, y)$, then from the analyticity of $n \wedge \nabla \wedge \boldsymbol{H}$ on $S$ it follows that $\partial H_{x} / \partial y, \partial H_{y} / \partial x$, $\partial H_{z} / \partial y$, and therefore that $H_{x}, H_{y}, H_{z}$ are all analytic on $S$. Therefore $\boldsymbol{H}$ and, from the Maxwell's equations, $E$ are analytic on $S$.

Acknowledgement. The research reported in this paper was sponsored by Electronic System Division AFSC, USAF, under Contract AF 19(628)-4884.

\section{References}

1. KLINE, M., \& I.W. KAY, Electromagnetic Theory and Geometrical Optics, New York: Interscience Publishers 1965.

2. Stratton, J.A., Electromagnetic Theory. New York: McGraw-Hill Book Company 1941.

3. WeYL, H., Ausbreitung elektromagnetischer Wellen über einem ebenen Leiter. Ann. Physik Ser., 4, 60, 481-500 (1919).

4. Wilcox, C.H., An expansion theorem for electromagnetic fields. Comm. Pure Appl. Math., 9 , 115- 134 (1956).

\section{Department of Electrical Engineering}

University of Michigan

Ann Arbor 